\title{
Managing Platforms and Ecosystems
}

\author{
Kaisa Still \\ VTT, Finland \\ kaisa.still@vtt.fi
}

\author{
Martha Russell \\ mediaX, Stanford \\ martha.russell@stanford.edu
}

\author{
Rahul Basole \\ Tennenbaum Inst., Georgia Tech \\ basole@gatech.edu
}

\begin{abstract}
This minitrack presents ten (10) papers that answered the call for perspectives for managing platforms and ecosystems. The papers address many management activities (designing, planning, implementing, decision making, and evaluating), from multiple perspectives (information systems, strategy, marketing, operations, computer science, social sciences, and policy). The papers continue and contribute to the scholarly exploration of concepts, theories, and tools.
\end{abstract}

\section{Introduction to the minitrack}

This minitrack follows our first HICSS minitrack at HICSS-49, which concentrated on 'Analytics and Decision Support for Ecosystems.' With the feedback from participants, the organizers as well as from the general disruptions taking place in the marketplace (for example with the successes of platform-based businesses such as Uber and AirBnB), we now want to focus on 'Managing platforms and ecosystems'.

'You cannot do it alone' and 'you do not have to do it alone' describe realities of the global interconnected economy. Contemporary organizations are struggling with managing the associated complexities. To support understanding and acting in this interconnected, even hyper-connected economies, the following concepts have been used:

- Ecosystems are complex socio, economic-, and technical systems that can be characterized by human networks that generate productive output on a sustainable basis, and as business ecosystems consisting of interdependent firms that form symbiotic relationships to create and deliver products and services.

- Platforms, conceptual or technological constructs to structure the relationships, provide a context for connections and value creation in an ecosystem. In practice digital platforms amplify the volume of opportunity in scaling toward success, allowing for multi-sided markets to emerge.
Conceptually, the mindsets, organizational constructs and the technological systems of multi-sided markets, platforms and ecosystems require a reframed perspective on strategic management that goes beyond extant literature on strategic and industrial innovation. While principles and tactics for managing platforms and ecosystems are emerging, more cross-disciplinary thought leadership and experience sharing are needed.

\section{Process toward minitrack}

For the minitrack call, we specified topics of interest to include, but not be limited to:

- Theories, models, and empirical studies of platforms and ecosystems

- Genesis, dynamics, and evolution of platforms and ecosystems

- Value-creation and enablement in ecosystems

- Platform and ecosystem business models

- Industry, technology, and investment platforms

- Technological and competitive disruption in ecosystems

- Analytics, visualization, and decision support for platform and ecosystems

- Best practices in platform and ecosystem management.

- Orchestration strategies of platforms and ecosystem

We were very happy to receive 21 papers by US, European and Asian researchers, which shows the global relevance and importance of this topic. The papers received addressed ecosystems (further defined as innovation ecosystems, digital ecosystems, service ecosystems and business ecosystems), and platforms (digital platforms, multi-sided platforms, engagement platforms)-some even talked about platform ecosystems. Some of the papers were case-studies, however, the use of quantitative approaches such as simulations and statistical analysis were dominating.

With the support of over 40 outstanding reviewers, we were able to choose the ten (10) papers to be accepted to the minitrack. We would like to thank the 
reviewers for their invaluable comments, which inspired both the authors as well as the organizers.

\section{The minitrack program}

We would like to thank the authors for responding to the comments from the reviewers, and for their subsequent modifications to the papers.

The ten (10) accepted papers will be presented in two sessions of the minitrack. Please note that interactions and discussions are a relevant part of the minitrack sessions.

\subsection{The first session addresses ecosystems}

1. Innovation Ecosystems vs. Innovation Systems in Terms of Collaboration and Co-creation of Value (Nataliya Smorodinskaya, Martha Russell, Daniel Katukov, Kaisa Still)

2. Emergence and dissemination of ecosystem concept in innovation studies: A systematic literature review study (Arash Hajikhani)

3. Shaping the Boundaries of a Service Ecosystem: The Case of Udacity (Hannes Rothe, Florian Steier)

4. Explaining Digital Ecosystem Evolution: The Revelatory Case of a Digital Platform Start-up in the 3D Printing Industry (Tyge-F. Kummer, Frederik von Briel)

\subsection{The second session addresses platforms}

5. Value-Creating Dynamics in Platform Ecosystem: A Firm Theory Lens (Dohoon Kim)

6. Uncovering the Nature of Platform-based Business Models: An Empirical Taxonomy (Karl Täucher, Sven M. Laudien)

7. The Antecedents of Transaction Costs in Digital Ecosystems: A Configurational View on the Interplay of App Architecture and Platform Governance (Dominik Dellerman, Fabian Rech)

8. Explaining standard dominance: The effect of influential network positions and structural holes (Geerten van de Kaa)
9. Too Big to Fail? Overcrowding a Multi-Sided Platform and Sustained Competitive Advantage (Pontus Huotari)

10. Visualizing the Geography of Platform Boundary Resources: The Case of the Global API Ecosystem (Jukka Huhtamäki, Rahul Basole, Kaisa Still, Martha Russell, Marko Turpeinen)

\section{For the presenters}

Please prepare a $\mathbf{1 5}$ minute presentation of your paper with the following components, while noting that HICSS suggests the use of 4 or 5 PowerPoint slides or transparencies with several bullet points on each: (1) Your/team introduction with relevant background info, and (2) The message of your paper: convey the main concepts, key results, implications, and future work.

Allow for a $\mathbf{1 5}$ minute discussion after your presentation. Note that discussion following presentation is a very valuable feature of a HICSS conference. The discussion is intended to connect your presentation to the theme of the session, so your comments and insights to addressing those are valued.

You are responsible for bringing your own computer. However, bringing a usb as a backup is recommended. Do not use time allocated to other presenters by going over your time. Please honor the 5minute warning given before the end of your allotted time.

\section{You are warmly welcome!}

Please join us for exciting presentations and discussions in the workshop! We are looking forward to the presenter insights, both theoretical as well as practical, into managing platforms and ecosystems - as well as your contributions to the discussions! 